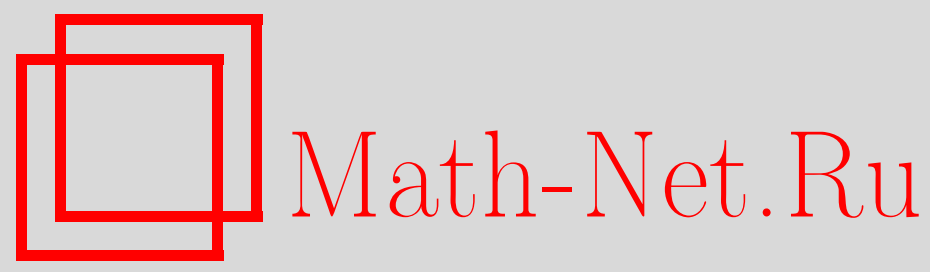

Г. М. Устинов, О подпространствах существования в $C(Q)$, Матем. заметки, 1999, том 65, выпуск 5, 726-737

DOI: https://doi.org/10.4213/mzm1104

Использование Общероссийского математического портала Math-Net.Ru подразумевает, что вы прочитали и согласны с пользовательским соглашением http://www.mathnet.ru/rus/agreement

Параметры загрузки:

IP : 54.89 .56 .158

26 апреля 2023 г., $15: 44: 46$

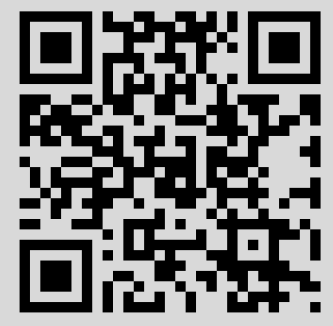




\section{О ПОДПРОСТРАНСТВАХ СУЩЕСТВОВАНИЯ В $C(Q)$}

\section{Г.М. Устинов}

Свойство пространства быть подпространством существования исследуется для тех подпространств $E \subset C(Q)$, для которых либо $E$, либо $C(Q) / E$ есть пространство Линденштраусса.

Для чебышевского подпространства $L \subset C(Q)$ приводится формула, в которой, используя аннулятор $L^{\perp}$, дается аналитическое представление ближайшего элемента.

Библиография: 13 названий.

Аппроксимационные свойства подпространств $E \subset C(Q), \operatorname{dim} E=\operatorname{codim} E=+\infty$, мало изучены, и формулируемьй различными авторами вопрос о том, содержит ли сепарабельное пространство $C(Q)$ чебьшевское подпространство $E, \operatorname{dim} E=\operatorname{codim} E=$ $+\infty$, остается открытым. Полученные в заметке конкретные результаты относятся к подпространствам $E$, для которых либо $E$, либо $C(Q) / E$ есть пространство Линденштраусса. В теореме 1 дается критерий подпространства существования $E \subset C(Q)$, если $C(Q) / E$ есть пространство $A(K)$ аффинных непрерьвных функций на компактном симплексе Шоке. Приводится критерий чебышевского подпространства, дающий "развернутое" представление ближайшего элемента (см. следствие 2, следствие 4). Из него вьводится, в частности, что если компакт $Q$ счетен, то никакое подпространство $E \subset C(Q), 1 \in E$, для которого $C(Q) / E$ есть пространство Линденштраусса, не будет чебьшевским.

Приведем необходимые сведения и обозначения.

Замкнутое подпространство $E$ банахова пространства $Z$ называется подпространством существования (пишем $E \in \mathcal{E}_{Z}$, а если $Z=C(Q)$, то пишем $E \in \mathcal{E}$ ), если для любого $z \in Z$ множество $P_{E} z=\left\{y \in E:\|y-z\|=\rho(z, E)=\inf _{v \in E}\|z-v\|\right\}$ не пусто. Чебышевским называется подпространство $E \in \mathcal{E}_{Z}$, для которого множество $P_{E} z$ одноточечно для любого $z \in Z$. Если банаховы пространства $Y, Z$ линейно изометричны, то пишем $Y \doteq Z$. Банахово пространство $Z$ называется пространством Линденштраyсcа, если $Z^{*} \doteq L^{1}(\mu)$ (см. [1]). Важным представителем этого класса пространств являются пространства $A(K)$ аффинных непрерывных функций, определенных на компактном симплексе Шоке $K$. Основные свойства пространств $A(K)$ и симплексов $K$ освещены в [2]. Заметим, что если $Q$ - компакт, то $C(Q) \doteq A(K)$, где $K=\left\{\varphi \in C^{*}(Q)\right.$ : $\|\varphi\|=1, \varphi \geq 0\}-w^{*}$-компактный симплекс Шоке. Если $U$ - компактное выпуклое множество, то $\partial U$ - совокупность экстремальных точек $U ; F_{\varphi}$ - наименьшая грань $U$, содержащая элемент $\varphi \in U$. Для банахова пространства $Z B_{Z}=\{z \in Z:\|z\| \leq 1\}$, $S_{Z}=\{z \in Z:\|z\|=1\}, P_{Z}=\left\{\varphi \in Z^{*}: \exists z \in S_{Z} ; \varphi(z)=\|\varphi\|\right\}$. Семейство 
функционалов $\left(\varphi_{\alpha}\right)_{\alpha \in A}, \varphi_{\alpha} \in Z^{*}$, назьвается тотальным на $Z$, если из $\varphi_{\alpha}(z)=0$ $\forall \alpha \in A$ следует $z=0$. Если $y \in Z$, то $\hat{y}$ - функционал, порожденньй элементом $y$ : $\hat{y}(\varphi)=\varphi(y) \forall \varphi \in Z^{*} ; Q$ - метризуемьй компакт, $C^{*}(Q)$ отождествляется с пространством всех регулярных счетно адлитивных мер $\mu$ ограниченной вариации, определенных на $\sigma$-алгебре $\Sigma$ борелевских подмножеств $е \subset Q$. Если $\varphi \in C^{*}(Q)$, то $\varphi^{+}\left(\varphi^{-}\right)$- положительная (отрицательная) составляющая при разложении меры $\varphi$ в смысле Хана (см. [3, теорема 10, с. 144]), $|\varphi|=\varphi^{+}+\varphi^{-}-$вариация меры $\varphi, \operatorname{supp} \varphi-$ пересечение всех замкнутых множеств $F_{\alpha}$, для каждого из которых $|\varphi|\left(Q \backslash F_{\alpha}\right)=0$.

Пусть $T=\bar{T} \subset Q$ и $I_{T}=\left\{f \in C(Q):\left.f\right|_{T}=0\right\}$. Тогда идеал $I_{T}$ является классическим примером нерефлексивного подпространства сушествования в $C(Q)$. Заметим, что $C(Q) / I_{T} \doteq C(T)$ - пространство Линденштраусса. Далее в теореме 1 рассмотрен более общий случай.

Лемма 1. Пусть $E \in \mathcal{E}, C(Q) / E=Y$ - пространство Линденштраусса. Тогда

1) $\partial B_{E \perp} \subset P_{C(Q)}$;

2) $\operatorname{supp} \varphi_{1} \cap \operatorname{supp} \varphi_{2}=\varnothing$, ecлu $\varphi_{1} \neq \pm \varphi_{2}, \quad \varphi_{i} \in \partial B_{E^{\perp}}$.

ДокАЗАтЕльство. Так как $E^{\perp} \doteq L^{1}(\mu)$, мы отождествим $E^{\perp}$ с $L^{1}(\mu)$ и для $\varphi \in$ $\partial B_{L^{1}(\mu)}$ определим множество $A=\{t: \varphi(t) \neq 0\}$. Если допустить, что найдется множество $е \subset A, 0<\mu(e)<\mu(A)$, то $\varphi=\left\|\tilde{\varphi}_{1}\right\| \cdot \varphi_{1}+\left\|\tilde{\varphi}_{2}\right\| \cdot \varphi_{2}$, где $\tilde{\varphi}_{1}=\varphi \cdot \chi_{e}, \tilde{\varphi}_{2}=\varphi \cdot \chi_{A \backslash e}$, $\varphi_{1}=\tilde{\varphi}_{1} /\left\|\tilde{\varphi}_{1}\right\|, \varphi_{2}=\tilde{\varphi}_{2} /\left\|\tilde{\varphi}_{2}\right\|, \chi_{s}-$ характеристическая функция множества $s \in \Sigma$. Поскольку $\varphi \in \partial B_{L^{1}(\mu)}$, получим противоречие, и потому $A$ - атом меры $\mu$. Значит, если $\varphi_{1}, \varphi_{2} \in \partial B_{L^{1}(\mu)}, \varphi_{1} \neq \pm \varphi_{2}$, то $F=\operatorname{co}\left(\varphi_{1} \cup \varphi_{2}\right)$ - грань $B_{L^{1}(\mu)}$. Для доказательства свойства 1$)$ возьмем $\varphi_{0} \in \partial B_{E} \perp$ и определим многозначное отображение

$$
\tau: B_{E^{\perp}} \rightarrow 2^{R}, \quad \tau(\varphi)= \begin{cases}1, & \varphi=\varphi_{0} ; \\ -1, & \varphi=-\varphi_{0} ; \\ |r| \leq 1, & \varphi \in B_{E^{\perp}}, \quad \varphi= \pm \varphi_{0} .\end{cases}
$$

Легко проверить, что выполнены все условия теоремы о селекции [1, с. 224], а потому найдется $y \in Y:\|y\|=1, y\left(\varphi_{0}\right)=1$. Так как $E \in \mathcal{E}$, найдется $g \in C(Q):\|g\|=1$, $g\left(\varphi_{0}\right)=1$. Отсюда следует, что $\varphi_{0} \in P_{C(Q)}$ и свойство 1) доказано.

Если $\varphi_{1}, \varphi_{2} \in \partial B_{E \perp}, \varphi_{1} \neq \pm \varphi_{2}$, то на грани $F$ определим две $w^{*}$-непрерьвные аффинные функции $h_{i}: h_{i}\left(\varphi_{1}\right)=1, h_{i}\left(\varphi_{2}\right)=(-1)^{i}$ и многозначные отображения $\tau_{i}: B_{E \perp} \rightarrow 2^{R}$,

$$
\tau_{i}(\varphi)=\left\{\begin{array}{lll}
\alpha+(-1)^{i}(1-\alpha), & \text { если } \varphi=\alpha \varphi_{1}+(1-\alpha) \varphi_{2}, \quad 0 \leq \alpha \leq 1 ; \\
-\left(\alpha+(-1)^{i}(1-\alpha)\right), & \text { если } \varphi=-\left(\alpha \varphi_{1}+(1-\alpha)\right) \varphi_{2}, \\
& & 0 \leq \alpha \leq 1, \quad i=1,2 ; \\
|r| \leq 1, & \text { если } \varphi \in B_{E^{\perp}} \backslash(F \cup(-F)) .
\end{array}\right.
$$

Применяя теорему о селекции $\left[1\right.$, с.224], найдем в $Y$ элементы $y_{1}, y_{2},\left\|y_{i}\right\|=1$, $y_{i}\left(\varphi_{1}\right)=1, y_{i}\left(\varphi_{2}\right)=(-1)^{i}, i=1,2$. Так как $E \in \mathcal{E}$, найдутся функции $g_{i} \in C(Q)$, $\left\|g_{i}\right\|=1, g_{i}\left(\varphi_{1}\right)=1, g_{i}\left(\varphi_{2}\right)=(-1)^{i}$. Поскольку $\left.g_{i}\right|_{\operatorname{supp} \varphi_{1}}=1,\left.g_{i}\right|_{\operatorname{supp} \varphi_{2}}=(-1)^{i}$, то $\operatorname{supp} \varphi_{1} \cap \operatorname{supp} \varphi_{2}=\varnothing$ и свойство 2) доказано. Лемма доказана.

ЗАМЕчАНИЕ 1. Из рассуждений, приведенных при доказательстве второй части леммы 1 , следует, что если $\varphi_{i} \in \partial B_{E^{\perp}}, \varphi_{1} \neq \pm \varphi_{2}$, то $\left|\varphi_{1}\right| \wedge\left|\varphi_{2}\right|=0$ (т.е. $\varphi_{1}, \varphi_{2}-$ дизъюнктные меры).

На следующее утверждение неоднократно ссылаемся далее. 
ПреДЛОЖЕНИЕ А. Для того чтобы $E \in \mathcal{E}_{Z}$, необходимо и достаточно, чтобы для каждого $w^{*}$-непрерывного функиионала $\varphi$, заданного на $E^{\perp}$, нашелся $w^{*}$-непрерывный функиионал $\Phi$, определенный на $Z^{*},\left.\Phi\right|_{E^{\perp}}=\varphi,\|\Phi\|=\|\varphi\|$. При әтом әлемент $y \in Z \backslash E$ имеет в $E$ единственный ближсайший әлемент тогда и только тогда, когда для функционала $\left.\hat{y}\right|_{E^{\perp}}$ найдется единственное $w^{*}$-непрерывное продолжение $\Phi$ на $Z^{*},\|\varphi\|=\left.\hat{y}\right|_{E^{\perp}} \|[4]$.

Это предложение есть обобщение на общий случай аналогичного утверждения, доказанного А. Л. Гаркави [5] для фактор-рефлексивных подпространств $E$.

Заметим, что для любого $w^{*}$-непрерьвного функционала $\Phi$, определенного на $Z^{*}$, найдется единственный элемент $z \in Z: \Phi(\varphi)=\varphi(z) \quad \forall \varphi \in Z^{*},\|\Phi\|=\|z\|$ (см. [3, теорема 9, с. 456]). Если $Z=C(Q), q \in Q$, то полагаем $\Phi(q)=\Phi\left(\delta_{q}\right), \delta_{q}(g)=g(q)$, $q \in C(Q)$.

Пусть $E \subset C(Q), \operatorname{supp} \varphi_{1} \cap \operatorname{supp} \varphi_{2}=\varnothing$, если $\varphi_{1} \neq \pm \varphi_{2}, \varphi_{i} \in \partial B_{E^{\perp}} ;$ Д $_{E}=$ $\bigcup_{\varphi \in \partial B_{E} \perp} \operatorname{supp} \varphi$ и определим отображение $\tau_{E}: Д_{E} \rightarrow \partial B_{E \perp}, \tau_{E}(q)=\varphi$, если $q \in$ $\operatorname{supp} \varphi$. Далее $\rho_{1}$ - метрика, определяющая $w^{*}$-топологию $B_{E^{\perp}}$.

Tеорема 1. Пусть $E \subset C(Q), C(Q) / E \doteq A(K)$, причем

1) грань $F_{\varphi}$ конечномерна $\forall \varphi \in \overline{\partial K} \backslash \partial K$,

2) $\partial F_{\varphi_{1}} \cap \partial F_{\varphi_{2}}=\varnothing$, eслu $\varphi_{1} \neq \varphi_{2}, \quad \varphi_{i} \in \overline{\partial K} \backslash \partial K$.

Для того чтобь $E \in \mathcal{E}$, необходимо и достаточно, чтобы выполнялись условия:

1) $\varphi \in P_{C(Q)} \forall \varphi \in \partial K$

2) $\operatorname{supp} \varphi_{1} \cap \operatorname{supp} \varphi_{2}=\varnothing$, если $\varphi_{i} \in \partial K, \varphi_{1} \neq \varphi_{2}$;

3) отображение $\tau_{E}$ равномерно непрерывно;

4) $\inf _{\varphi, \psi \in \partial K} \rho_{1}\left(\operatorname{supp} \varphi^{+}, \operatorname{supp} \psi^{-}\right)>0$.

ДокаЗАТЕЛЬСтво. НЕОБХодимость. Необходимость условий 1), 2) следует из леммы 1. Докажем необходимость условия 3$)$. Допустим, что $\rho\left(q_{k}^{1}, q_{k}^{2}\right) \rightarrow 0$, но $\inf _{k} \rho_{1}\left(\varphi_{k}^{1}, \varphi_{k}^{2}\right) \geq \varepsilon>0, \varphi_{k}^{i}=\tau_{E}\left(q_{k}^{i}\right)$. Не теряя общности, считаем, что $\varphi_{k}^{i} \rightarrow \varphi_{i}$. Возможны случаи:

a) $\varphi_{i} \in \partial K, i=1,2$

б) $\varphi_{1} \in \overline{\partial K} \backslash \partial K, \varphi_{2} \in \partial K$;

в) $\varphi_{i} \notin \overline{\partial K} \backslash \partial K$.

Разберем случай в). Допустим

$$
\varphi_{i}=\sum_{s=1}^{r_{i}} \alpha_{s}^{i} \psi_{s}^{i}, \psi_{s}^{i} \in \partial K, \quad \sum_{s=1}^{r_{i}} \alpha_{s}^{i}=1, \quad \alpha_{s}^{i}>0 .
$$

Пусть

$$
\left(\left(\bigcup_{k>p_{1}} \varphi_{k}^{1}\right) \cup\left(\bigcup_{s=1}^{r_{1}} \psi_{s}^{1}\right)\right) \cap\left(\left(\bigcup_{k>p_{2}} \varphi_{k}^{2}\right) \cup\left(\bigcup_{s=1}^{r_{2}} \psi_{s}^{2}\right)\right)=\varnothing
$$

и

$$
F_{i}=\overline{\mathrm{co}}\left(\left(\bigcup_{k>p_{i}} \varphi_{k}^{i}\right) \cup\left(\bigcup_{s=1}^{r_{i}} \psi_{s}^{i}\right)\right) .
$$

Тогда для любой точки $q \in \overline{\partial F_{i}} \backslash \partial F_{i}$ представляющая мера сосредоточена на $F_{i}$, а потому $F_{i}$ - замкнутая грань $K$ [6]. Так как $F_{1} \cap F_{2}=\varnothing$, по теореме 3.6 из [7] можно построить $h \in A(K),\|h\|=1,\left.h\right|_{F_{i}}=(-1)^{i}$. Так как $E \subset \mathcal{E}$, найдется $g \in C(Q)$, 
$\|g\|=1, g\left(\varphi_{k}^{i}\right)=(-1)^{i}$, а потому $\left.g\right|_{\operatorname{supp} \varphi_{k}^{i}}=(-1)^{i}$. Следовательно, $g\left(q_{k}^{1}\right)-g\left(q_{k}^{2}\right)=$ $-1-1=-2 \rightarrow 0$; получили противоречие. Аналогично разбираются случаи а), б), и необходимость условия 3 ) доказана. Докажем необходимость условия 4). Допустим, что $\rho\left(\operatorname{supp} \varphi_{k}^{+}, \operatorname{supp} \psi_{k}^{-}\right) \stackrel{k \rightarrow \infty}{\rightarrow} 0$. Не теряя обшности, считаем $\varphi_{k} \rightarrow \varphi, \psi_{k} \rightarrow \psi$ и разберем случаи: а) $\varphi=\psi$, б) $\varphi \neq \psi$.

Рассмотрим случай а) и допустим, что $\varphi \in \overline{\partial K} \backslash \partial K, \varphi=\sum_{s=1}^{r} \alpha_{s} \theta_{s}, \theta_{s} \in \partial K$, $\sum_{s=1}^{r} \alpha_{s}=1, \alpha_{s}>0$. Если $F=\overline{\text { со }}\left(\varphi \cup\left(\bigcup_{k=1}^{\infty} \varphi_{k}\right) \cup\left(\bigcup_{s=1}^{r} \theta_{s}\right) \cup\left(\bigcup_{k=1}^{\infty} \psi_{k}\right)\right)$, то (см. [6]), $F$ - замкнутая грань $K$ и по теореме 3.6 из [7] $A(K)$ содержит функцию $h,\|h\|=$ $1,\left.h\right|_{F} \equiv 1$. Так как $E \in \mathcal{E}$, найдется $g \in C(Q),\|g\|=1, g\left(\varphi_{k}\right)=g\left(\psi_{k}\right)=1$. Приходим к противоречию. Аналогично разбирается вариант, когда $\varphi \in \partial K$.

Случай б) разобран при доказательстве свойства 3 ). Необходимость доказана.

Достаточность. Пусть $G^{+}=\bigcup_{\varphi \in \partial K} \operatorname{supp} \varphi^{+}, G^{-}=\bigcup_{\varphi \in \partial K} \operatorname{supp} \varphi^{-}$; тогда по условию 4) $\bar{G}^{+} \cap \bar{G}^{-}=\varnothing$. Пусть далее $\bar{\tau}_{E}: \bar{G}=\bar{G}^{+} \cup \bar{G}^{-} \rightarrow \overline{\partial K}$ продолжение $\tau_{E}$ по непрерьвности. Возьмем определенный на $E^{\perp} w^{*}$-непрерьвньй функционал $g$ и определим функцию $\bar{g} \in C(\bar{G})$, полагая

$$
\bar{g}(q)= \begin{cases}g\left(\bar{\tau}_{E}(q),\right. & q \in \bar{G}^{+} \\ -g\left(\bar{\tau}_{E}(q),\right. & q \in \bar{G}^{-} .\end{cases}
$$

Пусть $g_{1} \in C(Q),\left.g_{1}\right|_{\bar{G}}=\bar{g},\left\|g_{1}\right\|=\left\|\overline{g_{1}}\right\|$. Легко убедиться, что $g_{1}$ определяет $w^{*}$-непрерьвное продолжение $g$ на все $C^{*}(\varphi): \hat{g_{1}}(\varphi)=\varphi\left(g_{1}\right), \varphi \in C^{*}(Q)$. Следовательно, $E \in \mathcal{E}$ по предложению А. Теорема доказана.

Приведем утверждение, позволяющее строить примеры подпространств, удовлетворяющих условиям теоремы 1.

ПрЕДЛОЖЕНИЕ В. Пусть $E \subset C(Q), E^{\perp} \doteq L^{1}(\mu)$. Тогда $C(Q) / E \doteq C(T)$ тогдa и только тогда, когда найдется такая функиия $h \in C(Q)$, что $\partial B_{E^{\perp}}=F \cup(-F)$, где $F=\bar{F}, F=\left\{\varphi \in \partial B_{E^{\perp}}: h(\varphi)=1\right\}$.

ДокАЗАтЕльство. Так как $(C(Q) / E)^{*} \doteq E^{\perp}$, справедливость предложения В следует из характеризации классов пространств Линденштраусса $[1$, c. 218].

ПримеР 1. Рассмотрим в $C^{*}([0 ; 1])$ подпространство $L$, порожденное мерами

$$
\begin{gathered}
\mu_{n}=-\frac{1}{n} \cdot \delta_{t_{n}}+\left(1-\frac{1}{n}\right) \cdot \delta_{s_{n}}, \quad t_{n}=\frac{1}{n+1}, \quad s_{n}=\left(1-\frac{1}{n+1}\right), \\
n=2,3, \ldots, \quad \mu_{1}=\delta_{t_{1}}, \quad t_{1}=1 .
\end{gathered}
$$

Тогда несложно убедиться в том, что $L-w^{*}$-замкнутое подпространство, $L \doteq l^{1}$. Пусть $E=L_{\perp}$. Очевидно, что найдется $a \in C([0 ; 1]), a\left(t_{n}\right)=-1, a\left(s_{n}\right)=1, n=2,3, \ldots$, $a\left(t_{1}\right)=1$. Так как $\mu_{n} \stackrel{w^{*}}{\longrightarrow} \mu_{1}$, по предложению В пространство $C([0 ; 1]) / E$ изометрично пространству $C$ сходящихся последовательностей. Из теоремы 1 следует, что $E \in \mathcal{E}$.

Кроме идеалов другими известными примерами нерефлексивных подпространств сушествования в $C(Q)$ являются подпространства Стоуна-Вейерштрасса [8]. Пусть $V-$ некоторый компакт, $\tau$ - непрерывное отображение $Q$ на $V$. Тогда подпространством Стоуна-Вейеритрасса называется подпространство $E=\{f: f(q)=g(\tau(q)), q \in$ $Q, g \in C(V)\}$. Ясно, что $1 \in E, E \doteq C(V), E$ не разделяет точки $Q$. Рассмотрим "дополнительньй" случай. 
ПрЕДЛОЖЕНИЕ 1. Пусть Е разделяет точки $Q, 1 \in E, E \doteq C\left(Q_{1}\right)$. Тогда найдутся однозначно определенные мнохество $T=\bar{T} \subset Q$, абстрактная функция $m: Q \backslash T \rightarrow C^{*}(T)$ maкuе, чmо $E=\{h \in C(Q): h(q)=m(q)(h)$, $q \in Q \backslash T\}$. При этом, если $Q \backslash T$ счетно $u\left|m\left(q_{1}\right)\right| \wedge\left|m\left(q_{2}\right)\right|=0, q_{1} \neq q_{2}$, то $C(Q) / E$ - пространство Линденштраусса.

ДоказАТЕЛЬСтво. Пусть $T=\left\{q \in Q: \delta_{\left.q\right|_{E}} \in \partial B_{E^{*}}\right\}$; тогда $T=\bar{T}, T$ гомеоморфно $Q_{1}$. Для каждого $q \in Q \backslash T$ функционал $\delta_{\left.q\right|_{E}} \notin \partial B_{E^{*}}$ и по теореме Рисса [5, теорема 3, с. 288] найдется однозначно определенная мера $\mu_{q} \in C^{*}(T), \mu_{q} \geq 0, \mu_{q}(1)=1$, $f(q)=\mu_{q}(f)$ для $f \in E$. Определим абстрактную функцию $m: Q \backslash T \rightarrow C^{*}(T)$, полагая $m(q)=\mu_{q}$. Пусть $L=\{h \in C(Q): h(q)=m(q)(h), q \in Q \backslash T\}$. Из определения $T, \varphi$ следует, что $E \subset L$. Для $\mu \in B_{E^{\perp}}$ определим меры $\mu_{1}, \mu_{2}$ :

$$
\mu_{1}(e)=\left\{\begin{array}{ll}
\mu(e), & e \subset T ; \\
0, & e \subset Q \backslash T ;
\end{array} \quad \mu_{2}(e)=\left\{\begin{array}{ll}
0, & e \subset T ; \\
\mu(e), & e \subset Q \backslash T ;
\end{array} \quad e \in \Sigma .\right.\right.
$$

Так как множество сужений на $T$ функций из $E$ совпадает с $C(T)$, то $\mu_{2} \neq 0$.

Для $q \in Q \backslash T$ полагаем $p_{q}=\left(\delta_{q}-\mu_{q}\right) / 2$. Покажем, что $p_{q} \in \partial B_{E^{\perp}}$. Допустим, что $\nu_{1}, \nu_{2} \in B_{E^{\perp}}, 0<\alpha<1, p_{q}=\alpha \nu_{1}+(1-\alpha) \nu_{2}$. Определим на $Q$ измеримую функцию

$$
r(t)= \begin{cases}1, & t=q \\ -1, & t \in \operatorname{supp} \mu_{q} \\ 0, & t \notin \operatorname{supp} p_{q} .\end{cases}
$$

Тогда $p_{q}(r)=1=\alpha \nu_{1}(r)+(1-\alpha) \nu_{2}(r)$, и потому $\nu_{1}(r)=\nu_{2}(r)=1$. Следовательно, $\nu_{i}(e)=0 \forall e \in \Sigma, e \subset Q \backslash \operatorname{supp} p_{q}, i=1,2$. Пусть $\nu_{i}\{q\}=\gamma_{i}, \tilde{\nu}_{i}=\gamma_{i} \delta_{q}-\gamma_{i} \mu_{q}$. Тогда $\nu_{i}-\tilde{\nu}_{i} \in E^{\perp}, \operatorname{supp}\left(\nu_{i}-\tilde{\nu}_{i}\right) \subset T$, что возможно лишь в случае $\nu_{i}=\tilde{\nu}_{i}$. Из равенств $\left\|\tilde{\nu}_{i}\right\|=\left|\gamma_{i}\right|\left\|\delta_{q}\right\|+\left|\gamma_{i}\right|\left\|\mu_{q}\right\|=2\left|\gamma_{i}\right|=1$ следует, что $\left|\gamma_{i}\right|=1 / 2, i=1,2$. Отсюда уже легко вьвести, что $\nu_{i}=p_{q}, i=1,2$, и потому $p_{q} \in \partial B_{E} \perp, q \in Q \backslash T$.

Для $\mu \in B_{E^{\perp}}, \mu=\mu_{1}+\mu_{2}, \mu_{2} \neq 0$, найдем последовательность мер $\nu_{n}=\sum_{i=1}^{k_{n}} \alpha_{i}^{n} \delta_{q_{i}^{n}}$, $q_{i}^{n} \in Q \backslash T, \sum_{i=1}^{k_{n}}\left|\alpha_{i}^{n}\right|=\left\|\mu_{2}\right\|, \nu_{n} \stackrel{w^{*}}{\longrightarrow} \mu_{2}$. Не теряя общности, можно считать, что

$$
\nu_{n}^{\prime}=2 \sum_{i=1}^{k_{n}} \alpha_{i}^{n} p_{q_{i}^{n}} \stackrel{w^{*}}{\rightarrow} \nu^{\prime}, \quad \nu_{n}^{\prime \prime}=\sum_{i=1}^{k_{n}} \alpha_{i}^{n} \mu_{q_{i}^{n}} \stackrel{w^{*}}{\longrightarrow} \nu^{\prime \prime}
$$

и потому $\mu_{2}=\nu^{\prime}+\nu^{\prime \prime}, \nu^{\prime} \in E^{\perp}, \operatorname{supp} \nu^{\prime \prime} \subset T$. Получаем $\mu=\mu_{1}+\nu^{\prime}+\nu^{\prime \prime}$, откуда следует, что $\mu_{1}+\nu^{\prime \prime} \in E^{\perp}$, а потому $\mu_{1}=-\nu^{\prime \prime}$. Итак, $\mu=\mu_{1}+\mu_{2}=-\nu^{\prime \prime}+$ $\nu^{\prime}+\nu^{\prime \prime}=\nu^{\prime}$. Следовательно, подпространство, порожденное мерами $p_{q}, q \in Q \backslash T$, $w^{*}$-плотно в $E^{\perp}$. Отсюда следует, что если $h \in L$, то $h \in E$, а потому $L=E$. Пусть теперь $Q \backslash T=\left\{q_{1}, q_{2}, \ldots, q_{n}, \ldots\right\}$. Возьмем $\mu \in B_{E \perp}$; тогда из приведенных ранее рассуждений следует, что $\mu=\sum_{n=1}^{\infty} \alpha_{n} p_{q_{n}}$. Так как меры $p_{q_{n}}$ взаимно дизъюнктны, нетрудно убедиться в том, что $\|\mu\|=\sum_{n=1}^{\infty}\left|\alpha_{n}\right|$, а потому $E^{\perp} \doteq l^{1}$. Предложение доказано.

СлЕДСТВИЕ 1. Пусть $Е \subset C(Q)$ удовлетворяет всем условиям предложения 1. Тогда $E \subset \mathcal{E}$ тогда и только тогда, когда $\forall h \in C(Q)$ найдется $g \in C(Q):\|g\|=$ $\sup _{q \in Q \backslash T}\left|p_{q}(g)\right|, g\left(p_{q}\right)=h\left(p_{q}\right) \quad \forall q \in Q \backslash T$. 
ЗАмЕчаниЕ 2. Пусть $Q$ - несчетньй компакт, $E \subset C(Q), 1 \in E, E$ разделяет точки $Q, E \doteq C\left(Q_{1}\right)$, где $Q_{1}$ - счетный компакт. Тогда $C(Q) / E$ не есть пространство Линденштраусса.

ДокАЗАтЕЛЬСтво. Допустим, что $E^{\perp} \doteq L^{1}(\mu)$. По замечанию 1 меры $\varphi_{1}, \varphi_{2}$ дизъюнктны, если $\varphi_{1} \neq \pm \varphi_{2}, \varphi_{i} \in \partial B_{E^{\perp}}$. По предложению 1 меры $p_{q}=$ $\left(\delta_{q}-\mu_{q}\right) / 2 \in \partial B_{E^{\perp}} \forall q \in Q \backslash T$. Так как $Q \backslash T$ несчетно, $T$ счетно, $\operatorname{supp} \mu_{q} \subset T$ $\forall q \in Q \backslash T$, получаем противоречие. Замечание доказано.

Пусть заданы $T=\bar{T} \subset Q, m: Q \backslash T \rightarrow C^{*}(T)$. Определим функцию

$$
\bar{m}(q)= \begin{cases}m(q), & q \in Q \backslash T ; \\ \delta_{q}, & q \in T \cap \overline{(Q \backslash T)} .\end{cases}
$$

Будем говорить, что функция $m w^{*}$-непрерывна, если $m\left(q_{i}\right) \rightarrow \bar{m}(q)$.

Легко установить справедливость следующего утверждения.

ПРЕДЛОЖЕНИЕ 1'. Пусть $T=\bar{T} \subset Q, m: Q \backslash T \rightarrow\left\{\mu \in C^{*}(T):\|\mu\|=1\right.$, $\mu \geq 0\}-w^{*}$-непрерывная функиия. Тогда $E=\{h \in C(Q): h(q)=m(q)(h)$, $q \in Q \backslash T\} \doteq C(T), \quad 1 \in E$.

Приведем пример подпространства $E \subset C(Q), E \doteq C\left(Q_{1}\right), 1 \in E, E \notin \mathcal{E}$.

ПримеР 2. В пространстве $\mathbb{R}^{1}$ с обычной метрикой рассмотрим множество $Q=$ $[0 ; 1 / 2] \cup\left\{t_{i}\right\}_{i=0}^{\infty} \cup\left\{s_{i}\right\}_{i=0}^{\infty}$, где $t_{i} \nearrow t_{0}, s_{i} \nearrow s_{0}, t_{1}>1 / 2, t_{0}=(2 / 3) s_{1}>2 / 3$, $s_{0}=1, T=[0 ; 1 / 2]$. Пусть далее

$$
\begin{aligned}
& \nu_{1}(f)=\frac{1}{2}\left[f\left(\frac{1}{8}\right)+f\left(\frac{1}{4}\right)\right], \quad \nu_{2}(f)=\frac{1}{2}\left[f\left(\frac{3}{8}\right)+f\left(\frac{1}{2}\right)\right], \\
& \nu_{i}(f)=\frac{i-1}{2 i}\left[f\left(\frac{1}{8}-\frac{1}{9^{i}}\right)+f\left(\frac{1}{4}-\frac{1}{9^{i}}\right)\right]+\frac{1}{i} f\left(\frac{1}{5^{2 i}}\right), \\
& u_{i}(f)=\frac{i-1}{2 i}\left[f\left(\frac{3}{8}-\frac{1}{9^{i}}\right)+f\left(\frac{1}{2}-\frac{1}{9^{i}}\right)\right]+\frac{1}{i} f\left(\frac{1}{5^{2 i+1}}\right), \quad i=1,2, \ldots .
\end{aligned}
$$

Определим функщию $m: Q \backslash T \rightarrow C_{\left[0 ; \frac{1}{2}\right]}^{*}$, полагая $m\left(t_{0}\right)=\nu_{1}, m\left(s_{0}\right)=\nu_{2}, m\left(t_{i}\right)=\nu_{i}$, $m\left(s_{i}\right)=u_{i}, i=1,2, \ldots$ По предложению $1^{\prime}$ подпространство $E=\{h \in C(Q): h(q)=$ $m(q)(h), q \in Q \backslash T\} \doteq C(T), 1 \in E$. Более того, легко проверить, используя предложение $\mathrm{B}$, что $C(Q) / E \doteq C(Q \backslash T)$. Также нетрудно убедиться, используя теорему 1 , что $E \notin \mathcal{E}$.

Введем класс подпространств $\mathcal{E}_{Z}^{\prime}$. Скажем, что $E \in \mathcal{E}_{Z}^{\prime}$, если $E \in \mathcal{E}_{Z}$ и найдется такое множество $\Phi_{E}=\left(\varphi_{\alpha}\right)_{\alpha \in A}, \varphi_{\alpha} \in Z^{*} \backslash E^{\perp}$, что 1) $E \cap \varphi_{\alpha}^{-1}(0)=E_{\alpha} \in \mathcal{E}_{Z} \forall \alpha \in A$, 2) $\Phi_{E}$ тотально на $Z$.

Пусть $\varphi \in Z^{*} \backslash E^{\perp}, L=E^{\perp} \oplus \operatorname{sp}(\varphi), y \in Z \backslash E, \rho(y, E)=d$. По теореме Хана-Банаха $[9$, теорема 4, с. 127$]$ продолжение $\tilde{y}$ на $L$ функционала $\left.\hat{y}\right|_{E^{\perp}}$ сохраняет норму тогда и только тогда, когда $\tilde{y}(\varphi) \in\left[\beta_{y}^{-}(\varphi), \beta_{y}^{+}(\varphi)\right]$, где

$$
\beta_{y}^{-}(\varphi)=\sup _{u \in E^{\perp}}\{\varphi(y)-d \cdot\|\varphi-u\|\}, \quad \beta_{y}^{+}(\varphi)=\inf _{u \in E^{\perp}}\{d \cdot\|u+\varphi\|-\varphi(y)\} .
$$


ПрЕДЛОЖЕНИЕ 2. Пусть $E \in \mathcal{E}_{Z}^{\prime}, y \notin E$. Для того чтобы y имел в $E$ единственный ближсайший әлемент, необходимо и достаточно, чтобы $\beta_{y}^{-}(\varphi)=\beta_{y}^{+}(\varphi)$ $\forall \varphi \in \Phi_{E}$.

ДоказАтЕльСтво. НЕоБХодимость. Допустим, что $\beta_{y}^{+}\left(\varphi_{0}\right)>\beta_{y}^{-}\left(\varphi_{0}\right)$ для $\varphi_{0} \in$ $\Phi_{E}$ и $L=E^{\perp} \oplus \mathrm{sp}\left(\varphi_{0}\right)$. Тогда на $L$ функционал $\left.\hat{y}\right|_{E^{\perp}}$ имеет два различных продолжения $\psi_{1}, \psi_{2},\left\|\psi_{1}\right\|=\left\|\psi_{2}\right\|=d$. Так как $E_{\varphi_{0}}=\left\{z \in E: \varphi_{0}(z)=0\right\} \in \mathcal{E}_{Z}$, по предложению А функционалы $\psi_{1}, \psi_{2}$ имеют $w^{*}$-непрерьвные продолжения $\tilde{\psi}_{1}, \tilde{\psi}_{2}$ на все $Z^{*}$, $\left\|\psi_{1}\right\|=\left\|\tilde{\psi}_{1}\right\|=\left\|\psi_{2}\right\|=\left\|\tilde{\psi}_{2}\right\|$. Окончательно получаем, что $\left.\hat{y}\right|_{E \perp}$ имеет два различных $w^{*}$-непрерьвных продолжения на $Z^{*}$ с сохранением нормы, а потому по предложению $\mathrm{A}$ множество $P_{E} y$ не одноточечно. Получили противоречие и необходимость доказана.

Достаточность. Если предположить, что $\left.\hat{y}\right|_{E \perp}$ имеет два $w^{*}$-непрерьвных продолжения $\psi_{1}, \psi_{2}$ на все $Z^{*},\left\|\psi_{1}\right\|=\left\|\psi_{2}\right\|=d$, то найдется $\varphi_{0} \in \Phi_{E}: \psi_{1}\left(\varphi_{0}\right) \neq \psi_{2}\left(\varphi_{0}\right)$. Следовательно, $\left.\hat{y}\right|_{E^{\perp}}$ имеет два различных продолжения на $L=E^{\perp} \oplus \operatorname{sp}\left(\varphi_{0}\right)$, а потому $\beta_{y}^{-}\left(\varphi_{0}\right)<\beta_{y}^{+}\left(\varphi_{0}\right)$. Полученное противоречие доказьвает достаточность.

Предложение доказано.

СлЕДСТВИЕ 2. Для того чтобы рефлексивное подпространство $E \subset C(Q)$ было чебышевским, необходимо и достаточно, чтоби: 1) $\left.\delta_{q} \notin E^{\perp} \forall q \in Q, 2\right) \beta_{g}^{-}\left(\delta_{q}\right)=$ $\beta_{g}^{+}\left(\delta_{q}\right) \quad \forall g \in C(Q) \backslash E, \forall q \in Q$. Ecли $h_{g}-$ ближсайший әлемент для функиии $g$, то $h_{g}(q)=g(q)-\beta_{g}^{+}\left(\delta_{q}\right), q \in Q$.

Пусть $\varkappa$-естественное вложение $Z$ в $Z^{* *}$ и $\widetilde{E}=\left(E^{\perp}\right)^{\perp}-w^{*}$-замыкание $\varkappa E$. Известно [10], что $\widetilde{E} \in \mathcal{E}_{Z^{* *}}$ и каждый элемент $\tilde{y} \in P_{\widetilde{E}}(\varkappa y)$ называется обобщенным әлементом наилучшего приближсения для $y \in Z \backslash E$ (см. [11]).

Естественно подпространство $E \subset Z$ называть обобщенным чебышевским, если каждьй элемент $\varkappa y, y \in Z \backslash E$, имеет единственньй обобщенный элемент наилучшего приближения.

СлЕДСТВИЕ 3. Пусть $Q=\left\{q_{1}, q_{2}, \ldots, q_{n}, \ldots\right\}-$ счетный компакт, $E \subset C(Q)$. Для того, чтобы Е было обобщенным чебышевским подпространством, необходимо и достаточно, чтобь: 1) $\left.\delta_{q_{i}} \notin E^{\perp} \forall q_{i} \in Q, 2\right) \quad \beta_{g}^{+}\left(\delta_{q_{i}}\right)=\beta_{g}^{-}\left(\delta_{q_{i}}\right) \forall g \in C(Q) \backslash E$.

ДоказАтельство. НЕОБХоДИмость. Допустим, что $\delta_{q_{i_{0}}} \in E^{\perp}$. Возьмем функцию $g_{0} \in E, 0<\left\|g_{0}\right\|<1$, и построим функцию $g \in C(Q),\|g\|=1, g\left(q_{i_{0}}\right)=1$, $\left\|g-g_{0}\right\|=1$. Тогда $\rho(g, E)=1, g_{0}, 0 \in P_{E} g$, и необходимость условия 1$)$ доказана. Известно, что $C^{* *} \doteq m$ где $m=\left\{\varphi=\left(\varphi_{1}, \varphi_{2}, \ldots, \varphi_{i}, \ldots\right),\|\varphi\|=\sup \left|\varphi_{i}\right|<+\infty\right\}$. Определим функционалы $\psi_{i}$ на $m$, полагая $\psi_{i}(\varphi)=\varphi_{i}, i=1,2, \ldots$ Пусть $e_{i}-$ орт естественного базиса пространства $\ell^{1}, \widetilde{E}_{i}=\widetilde{E} \cap \psi_{i}^{-1}(0)$. Тогда $\widetilde{E}_{i}=\left(E^{\perp} \oplus\left[e_{i}\right]\right)^{\perp}$, и потому $\widetilde{E}_{i} \in \mathcal{E}_{m}, i=1,2, \ldots$ Так как $\left(\psi_{i}\right)_{i=1}^{\infty}-$ тотально на $m$, то необходимость условия 2) следует из предложения 2. Достаточность получается из предложения 2. Следствие доказано.

Если $\nu \in C^{*}(Q)$, то $\nu=r_{t} \delta_{t}+\nu_{t}$, где $r_{t} \in \mathbb{R}, \nu_{t}$ - мера, сингулярная с $\delta_{t}$. Каждому $\varphi \in\left(E^{\perp}\right)^{*},\|\varphi\|=d$, сопоставим два множества

$$
\begin{aligned}
& \Phi_{\varphi}^{+}=\left\{\nu \in S_{E^{\perp}}: r_{t}<0, d-\varphi(\nu) \leq 2 d \cdot\left|r_{t}\right|\right\} \\
& \Phi_{\varphi}^{-}=\left\{\nu \in S_{E^{\perp}}: r_{t}>0, d-\varphi(\nu) \leq 2 d \cdot r_{t}\right\} .
\end{aligned}
$$


Лемма 2. Пусть $E \subset C(Q), \delta_{t} \notin E^{\perp}, \quad E_{1}=E^{\perp} \oplus \operatorname{sp}\left(\delta_{t}\right), \quad \varphi \in\left(E^{\perp}\right)^{*},\|\varphi\|=$ $d \neq 0, \quad \psi \in E_{1}^{*},\left.\quad \psi\right|_{E^{\perp}}=\varphi$. Тогда $\|\psi\|=d$ тогда и только тогда, когда $\psi\left(\delta_{t}\right) \in$ $\left[\beta_{\varphi}^{-}\left(\delta_{t}\right), \beta_{\varphi}^{+}\left(\delta_{t}\right)\right]$, əдe

$$
\begin{aligned}
& \beta_{\varphi}^{-}\left(\delta_{t}\right)=\left\{\begin{array}{lll}
-d, & \text { если } & \Phi_{\varphi}^{-}=\varnothing ; \\
\max \left(-d, d-\inf _{\nu \in \Phi_{\varphi}^{-}}\left\{\frac{d-\varphi(\nu)}{r_{t}}\right\}\right), & \text { если } & \Phi_{\varphi}^{-} \neq \varnothing ;
\end{array}\right. \\
& \beta_{\varphi}^{+}\left(\delta_{t}\right)=\left\{\begin{array}{lll}
d, & \text { если } & \Phi_{\varphi}^{+}=\varnothing ; \\
\min \left(d,-d+\inf _{\nu \in \Phi_{\varphi}^{+}}\left\{\frac{d-\varphi(\nu)}{\left|r_{t}\right|}\right\}\right), & \text { если } & \Phi_{\varphi}^{+} \neq \varnothing .
\end{array}\right.
\end{aligned}
$$

ДокАЗАТЕЛЬСТво. Пусть $\varphi_{1}=\varphi / d$. По формулам (1) получаем

$$
\beta_{\varphi}^{+}\left(\delta_{t}\right)=\inf _{\nu \in E^{\perp}}\left\{d \cdot\left\|\nu+\delta_{t}\right\|-\varphi(\nu)\right\}=d \cdot \inf _{\nu \in E^{\perp}}\left\{\left\|\nu+\delta_{t}\right\|-\varphi_{1}(\nu)\right\}=d \cdot \beta_{\varphi_{1}}^{+}\left(\delta_{t}\right) .
$$

Выведем формулы, соответствуюшие $\varphi_{1}$. Так как $\left\|\varphi_{1}\right\|=1$, то $\beta_{\varphi_{1}}^{+}\left(\delta_{t}\right) \leq 1, \beta_{\varphi_{1}}^{-}\left(\delta_{t}\right) \geq$ -1 . Пусть

$$
\begin{array}{ll}
C_{1}=\left\{\nu \in E^{\perp}: r_{t} \geq 0\right\}, & C_{2}=\left\{\nu \in E^{\perp}:-1<r_{t}<0\right\} \\
C_{3}=\left\{\nu \in E^{\perp}: r_{t} \leq-1\right\}, & C_{2}^{\prime}=C_{2} \cap S_{E^{\perp}}, \quad C_{3}^{\prime}=C_{3} \cap S_{E^{\perp}} .
\end{array}
$$

Тогда

$$
C_{2}=\bigcup_{\substack{\nu \in C_{2}^{\prime} \\ \nu=r_{t} \delta_{t}+\nu_{t}}}\left\{\lambda \nu: 0<\lambda<\frac{1}{\left|r_{t}\right|}\right\}, \quad C_{3}=\bigcup_{\substack{\nu \in C_{3}^{\prime} \\ \nu=r_{t} \delta_{t}+\nu_{t}}}\left\{\lambda \nu: \lambda \geq \frac{1}{\left|r_{t}\right|}\right\}
$$

Имеем $\beta_{\varphi_{1}}^{+}\left(\delta_{t}\right)=\min \left(a_{1}, a_{2}, a_{3}\right)$, где $a_{i}=\inf _{\nu \in C_{i}}\left\{\left\|\nu+\delta_{t}\right\|-\varphi_{1}(\nu)\right\}, i=1,2,3$;

$$
\begin{aligned}
a_{1} & =\inf _{\nu \in C_{1}}\left\{\left|r_{t}+1\right|+\left\|\nu_{t}\right\|-\varphi_{1}(\nu)\right\}=\inf _{\nu \in C_{1}}\left\{\|\nu\|-\varphi_{1}(\nu)\right\}+1=+1 ; \\
a_{2} & =\inf _{\nu \in C_{2}}\left\{\left\|\nu+\delta_{t}\right\|-\varphi_{1}(\nu)\right\}=\inf _{\nu \in C_{2}}\left\{\|\nu\|-\varphi_{1}(\nu)+\left|r_{t}+1\right|-r_{t}\right\} \\
& =\inf _{\nu \in C_{2}}\left\{\|\nu\|\left[1-\varphi_{1}\left(\frac{\nu}{\|\nu\|}\right)+\frac{2 r_{t}}{\|\nu\|}\right]+1\right\} .
\end{aligned}
$$

Зафиксируем $\nu \in C_{2}^{\prime}, \nu=r_{t} \cdot \delta_{t}+\nu_{t}$. Тогда

$$
\inf _{0<\lambda<\frac{1}{\left|r_{t}\right|}}\left\{\lambda\left[1-\varphi_{1}(\nu)+2 r_{t}\right]+1\right\}= \begin{cases}1, & \text { если } 1-\varphi(\nu)+2 r_{t} \geq 0 \\ -1+\frac{1-\varphi_{1}(\nu)}{\left|r_{t}\right|}, & \text { если } 1-\varphi(\nu)+2 r_{t}<0\end{cases}
$$

Следовательно,

$$
a_{2}=\min \left(1, \inf _{\nu \in C_{2}^{\prime} \cap \Phi_{\varphi_{1}}^{+}}\left\{\frac{1-\varphi(\nu)}{\left|r_{t}\right|}-1\right\}\right) .
$$


Далее,

$$
\begin{aligned}
a_{3} & =\inf _{\nu \in C_{3}}\left\{\left\|\nu+\delta_{t}\right\|-\varphi_{1}(\nu)\right\}=\inf _{\nu \in C_{3}}\left\{\left\|\nu_{t}\right\|+\left|r_{t}\right|-\varphi_{1}(\nu)+\left|r_{t}+1\right|-\left|r_{t}\right|\right\} \\
& =\inf _{\nu \in C_{3}}\left\{\|\nu\|\left(1-\varphi_{1}\left(\frac{\nu}{\|\nu\|}\right)\right)-1\right\}=\inf _{\nu \in C_{3}^{\prime}}\left\{\frac{1-\varphi_{1}(\nu)}{\left|r_{t}\right|}-1\right\}=\min \left(a_{3}^{\prime}, a_{3}^{\prime \prime}\right),
\end{aligned}
$$

где

$$
a_{3}^{\prime}=\inf _{\nu \in C_{3}^{\prime} \cap \Phi_{\varphi_{1}}^{+}}\left\{\frac{1-\varphi_{1}(\nu)}{\left|r_{t}\right|}-1\right\}, \quad a_{3}^{\prime \prime}=\inf _{\nu \in C_{3}^{\prime} \cap\left(S_{E^{\perp}} \backslash \Phi_{\varphi_{1}}^{+}\right)}\left\{\frac{1-\varphi_{1}(\nu)}{\left|r_{t}\right|}-1\right\} .
$$

Если $\nu \in C_{3}^{\prime} \cap\left(S_{E} \perp \backslash \Phi_{\varphi_{1}}^{+}\right)$, то $\left(1-\varphi_{1}(\nu)\right) /\left|r_{t}\right| \geq 2$, а потому $a_{3}^{\prime \prime} \geq 1$. Следовательно,

$$
a_{3}=\min \left(1, \inf _{\nu \in C_{3}^{\prime} \cap \Phi_{\varphi_{1}}^{+}}\left\{\frac{1-\varphi_{1}(\nu)}{\left|r_{t}\right|}-1\right\}\right)
$$

В результате получена формула для $\beta_{\varphi_{1}}^{+}\left(\delta_{t}\right)$. Аналогично вьводится формула для $\beta_{\varphi_{1}}^{-}\left(\delta_{t}\right)$. Общий случай легко получается из рассмотренного. Лемма доказана.

Лемма 3. Пусть $E \subset C(Q), \quad \delta_{q} \notin E^{\perp} \quad \forall q \in Q, \quad h \in C(Q) \backslash E, \quad \tilde{h}-w^{*}$-непрерывное продолжение $\left.\hat{h}\right|_{E^{\perp}}$ на все $C^{*}(Q)$. Тогда $\tilde{h}(q)$ есть непрерывнаая селекиия многозначного отображения $\tau: Q \rightarrow 2^{R}, \quad \tau(q)=\left[H^{-}(q), H^{+}(q)\right]$, əде $H^{-}(q)=\inf _{n} \sup _{t \in V_{n}(q)} \beta_{h}^{-}\left(\delta_{t}\right), \quad H^{+}(q)=\sup _{n} \inf _{t \in V_{n}(q)} \beta_{h}^{+}\left(\delta_{t}\right), \quad V_{n}(q)=$ $\left\{t \in Q: \rho(t, q)<\frac{1}{n}\right\}, \quad n=1,2, \ldots$

ДоказАтЕльСтво. Так как $\tilde{h}(t)=\tilde{h}\left(\delta_{t}\right) \in\left[\beta_{h}^{-}\left(\delta_{t}\right), \beta_{h}^{+}\left(\delta_{t}\right)\right]$, то $\tilde{h}(t) \leq \beta_{h}^{+}\left(\delta_{t}\right)$, и потому $\inf _{t \in V_{n}(q)} \tilde{h}(t) \leq \inf _{t \in V_{n}(q)} \beta_{h}^{+}\left(\delta_{t}\right), \quad \tilde{h}(q)=\sup _{n} \inf _{t \in V_{n}(q)} \tilde{h}(t) \leq$ $\sup _{n} \inf _{t \in V_{n}(q)} \beta_{h}^{+}\left(\delta_{t}\right)=H^{+}(q)$. Аналогично проверяется, что $\tilde{h}(q) \geq H^{-}(q)$. Лемма доказана.

Пусть $Q_{0}$ - множество изолированных точек $Q$.

Теорема 2. Пусть $Q=\bar{Q}_{0}, \quad 1 \in E$. Для того чтобы $E$ было чебышевским, необходимо и достаточно, чтобь $\forall h \in C(Q) \backslash E$ выполнялось: 1) $H^{+}(q)=H^{-}(q)$ $\forall q \in Q, 2) H^{+}(q)(\varphi)=\varphi(h) \quad \forall \varphi \in E^{\perp}$.

ДокаЗАТЕЛЬСтво. ДоСтаточность. Если $q_{i} \rightarrow q$, то легко проверить, что $\underline{\lim } H^{+}\left(q_{i}\right) \geq H^{+}(q)$ и $\varlimsup H^{-}\left(q_{i}\right) \leq H^{-}(q)$, и потому из условия 1$)$ следует, что $H(q)=$ $\overline{H^{+}}(q) \in C(Q)$. Условие 2) означает, что $\left.H\right|_{E^{\perp}}=\left.\hat{h}\right|_{E^{\perp}}$. Заметим далее, что если $q \in Q_{0}$, то $H(q)=\beta_{h}^{+}\left(\delta_{q}\right)=\beta_{h}^{-}\left(\delta_{q}\right)$, и потому $H^{+}(q) \leq \rho(h, E)$. Следовательно, $\left\|H^{+}\right\|=\|H\| \leq \rho(h, E)$. С другой стороны, так как $\left.H\right|_{E^{\perp}}=\left.\hat{h}\right|_{E^{\perp}}$, то $\|H\| \geq \rho(h, E)$. Окончательнополучаем $\|H\|=\rho(h, E)$ и функция $H$ есть $w^{*}$-непрерьвноепродолжение функционала $\left.\hat{h}\right|_{E^{\perp}}$, сохраняющее норму. Из предложения А следует, что $E \in \mathcal{E}$. Условие $\beta_{h}^{+}\left(\delta_{q}\right)=\beta_{h}^{-}\left(\delta_{q}\right)$ при $q \in Q_{0}$ гарантирует единственность этого $w^{*}$-непрерывного продолжения и потому $E$ - чебышевское подпространство. 
НЕоБходимость. Допустим, что $E$ - чебышевское подпространство; возьмем $h \in$ $C(Q) \backslash E,\|h\|=\rho(h, E)=d$ и пусть $\nu \in S_{E^{\perp}} \cap P_{C}, \nu(h)=d$. Тогда из формул (2), определяющих $\beta_{h}^{+}\left(\delta_{t}\right), \beta_{h}^{-}\left(\delta_{t}\right)$, следует, что $\beta_{h}^{+}\left(\delta_{t}\right)=\beta_{h}^{-}\left(\delta_{t}\right)$ при $t \in \operatorname{supp} \nu \cap Q_{0}$. B самом деле, пусть $\nu=r_{t} \cdot \delta_{t}+\nu_{t}$ и $r_{t}<0$. Тогда множество $\Phi^{+} \neq \varnothing$, ибо $\nu \in \Phi^{+}$ и $\beta_{h}^{+}\left(\delta_{t}\right)=-d$. Так как $-d \leq \beta_{h}^{-}\left(\delta_{t}\right) \leq \beta_{h}^{+}\left(\delta_{t}\right)$, то $\beta_{h}^{-}\left(\delta_{t}\right)=\beta_{h}^{+}\left(\delta_{t}\right)$. Аналогично разбирается случай, когда $r_{t}>0$.

Поскольку $H^{+}(q)=\beta_{h}^{+}\left(\delta_{q}\right), H^{-}(q)=\beta_{h}^{-}\left(\delta_{q}\right)$ при $q \in Q_{0}$, то $H^{+}(q)=H^{-}(q)$ при $q \in Q_{0} \cap \operatorname{supp} \nu$. Пусть $\hat{h}-w^{*}$-непрерьвное продолжение $\left.\hat{h}\right|_{E^{\perp}}$ на все $C^{*}(Q)$, $\|\hat{h}\|=d$. Тогда по лемме $3 \hat{h}(q) \leq H^{+}(q)$. Допустим, что $\hat{h}(q)<H^{+}(q)$ при некотором $q \in Q$. Тогда в силу полунепрерывности снизу функции $H^{+}(q)$ это неравенство вьполняется и в некоторой окрестности точки $q$. Следовательно, найдется $q_{1} \in Q_{0}: \tilde{h}\left(q_{1}\right)<$ $H^{+}\left(q_{1}\right)=\beta_{h}^{+}\left(\delta_{q_{1}}\right)$, причем в силу сказанного ранее, $q_{1} \notin \operatorname{supp} \nu$. Определим на $C^{*}(Q)$ $w^{*}$-непрерьвньй функционал $\Phi(\varphi)=\tilde{h}(\varphi)-a \cdot \varphi\left\{q_{1}\right\}, \varphi \in C^{*}(Q), a=d+\beta_{h}^{+}\left(\delta_{q_{1}}\right)$. Оценим $\sup _{\varphi \in S_{E \perp}} \Phi(\varphi)=d_{1}$. Рассмотрим два случая:

1) $\tilde{h}(\varphi) \geq 0, \varphi\left\{q_{1}\right\}<0$;

2) $\tilde{h}(\varphi) \geq 0, \varphi\left\{q_{1}\right\} \geq 0$.

В первом случае воспользуемся формулой $(2)$, определяющей $\beta_{h}^{+}\left(\delta_{q_{1}}\right)$; из нее следует неравенство $d-\tilde{h}(\varphi) \geq a\left|\varphi\left\{q_{1}\right\}\right|$ или $d \geq \Phi(\varphi)$. Так как $\left.\tilde{h}\right|_{E^{\perp}}=h$, то в первом случае $-d \leq \Phi(\varphi) \leq d$. Вовтором случае $\Phi(\varphi) \geq-2 d \cdot \varphi\left\{q_{1}\right\} \geq-d$, так как $\left|\varphi\left\{q_{1}\right\}\right| \leq 1 / 2$. Следовательно, и в этом случае $-d \leq \Phi(\varphi) \leq d$. В результате неравенства $-d \leq \Phi(\varphi) \leq d$ установлены, если $\varphi \in S_{E^{\perp}}, h(\varphi) \geq 0$. Отсюда следует, что $-d \leq \Phi(\varphi) \leq d \forall \varphi \in S_{E^{\perp}}$, а потому $d_{1} \leq d$. Так как $\Phi(\nu)=d$, то $d_{1}=d$. Если функционал $\tilde{h}$ порождается элементом $g \in C(Q)$, то функционал $\Phi(\varphi)$ порождается функцией $g_{1}(q)=g(q)-a \chi_{q_{1}}(q)$, где $\chi_{q_{1}}(q)$ - характеристическая функция одноточечного множества $\left\{q_{1}\right\}$. Ясно, что $g_{1} \in C(Q)$, и так как $\tilde{h}\left(\delta_{q_{1}}\right)<\beta_{h}^{+}\left(\delta_{q_{1}}\right)$, то $\left|g_{1}\left(q_{1}\right)\right|=\left|\tilde{h}\left(\delta_{q_{1}}\right)-a\right|>d$. Следовательно, $\left\|g_{1}\right\|>d$ и $\rho\left(g_{1}, E\right)=d$. Значит, $P_{E} g_{1}=\ell \neq 0$. Пусть $g_{1}=\ell+g_{0}$, где $g_{0} \in C(Q)$, $\left\|g_{0}\right\|=d$. Так как $g_{1}(\nu)=\Phi(\nu)=d$, то $\nu\left(g_{0}\right)=\left\|g_{0}\right\|=d$, а значит, $\left.g_{0}\right|_{\operatorname{supp} \nu}=\left.g\right|_{\operatorname{supp} \nu}$. Из равенства $g(q)-a \chi_{q_{1}}(q)=g_{0}(q)+\ell(q) \forall q \in Q$ следует, что $\left.\ell\right|_{\operatorname{supp} \nu}=0$. Следовательно, $E$ - не чебышевское подпространство по лемме С. Полученное противоречие показывает, что $\tilde{h}(q)=H^{+}(q)$. Аналогично доказьвается, что $\tilde{h}(q)=H^{-}(q) \forall q \in Q$. Следовательно, $H^{-}(q)=H^{+}(q)$ и необходимость условия 1) в этом случае доказана.

Переходя к общему случаю, заметим, что если $h \in C(Q) \backslash E, h=h_{0}+\ell, \ell \in E$, $\rho(h, E)=\left\|h_{0}\right\|$, то функционалы $\hat{h}, \hat{h}_{1}, h_{1}=h-\ell$ совпадают на $E^{\perp}$. Для элемента $h-\ell$ имеем $\|h-\ell\|=\rho(h-\ell, E)$, и для него необходимость уже доказана. Следовательно, для элемента $h$ имеем $H^{+}(q)=H^{-}(q) \forall q \in Q$ и необходимость условия 1) доказана.

Если $\tilde{h}-w^{*}$-непрерьвное продолжение на $C^{*}(Q)$ функционала $\left.\hat{h}\right|_{E^{\perp}},\|\tilde{h}\|=\left\|\left.\hat{h}\right|_{E^{\perp}}\right\|$, то по доказанному $H^{+}(q)=H^{-}(q)=\tilde{h}(q) \forall q \in Q$. Следовательно, $\forall \varphi \in E^{\perp}$ имеем: $\tilde{h}(\varphi)=\varphi(h)=H^{+}(q)(\varphi)$, и необходимость условия 2) доказана. Теорема доказана.

СледствИЕ 4. Если $Q=\bar{Q}_{0}, 1 \in E, E$ - чебышевское подпространство, $h \in C(Q) \backslash E$, mо $h(q)-H^{+}(q)$ есть ближайший элемент для функиии $h$.

Следующая лемма, вероятно, хорошо известна.

Лемма С. Пусть $E \subset \mathrm{C}(Q), \varphi \in E^{\perp} \cap P_{C(Q)} u E$ coдержит функиию $g, g \neq 0$, $\left.g\right|_{\operatorname{supp} \varphi}=0$. Тогда $E-$ не чебышевское подпространство.

Заметим, что более общий факт доказан в [12, теорема 7]. 
Лемма 4. Пусть $E \subset Z, z_{i} \in Z \backslash E, \quad i=1,2, \ldots, k, E_{1}=E \oplus \operatorname{sp}\left(z_{i}\right)_{i=1}^{k} . E c \Omega u$ $E \in \mathcal{E}, m o E_{1} \in \mathcal{E}$.

ДокАЗАТЕЛЬСТво. Пусть $\varphi-w^{*}$-непрерывный функционал, определенный

на $E_{1}^{\perp}$ и $\tilde{\varphi}$-продолжение $\varphi$ по теореме Хана-Банаха на $E^{\perp}$. Тогда легко проверить, что $\tilde{\varphi}-w^{*}$-непрерьвньй функционал на $E^{\perp}$. Так как $E \in \mathcal{E}$, по предложению А для функционала $\tilde{\varphi}$ найдется $w^{*}$-непрерывное продолжение $\widetilde{\varphi}$ на $Z^{*},\|\widetilde{\varphi}\|=\|\tilde{\varphi}\|=\|\varphi\|$ В итоге, по предложению $\mathrm{A} E_{1} \in \mathcal{E}$. Лемма доказана.

Лемма 5. Пусть $E \in \mathcal{E}, L \subset E^{\perp}, \operatorname{dim} E^{\perp} / L<+\infty$. Тогда $P_{C} \cap L$ всюду плотно в $L$.

ДокаЗАТЕЛЬСтво. Пусть $E_{1}=(L)_{\perp}, Y=C(Q) / E_{1}$ и возьмем $\varphi \in P_{Y},\|\varphi\|=1$. Следовательно, для некоторого $y \in Y,\|y\|=1$, имеем $\varphi(y)=1$. Пусть $\tau$ - естественное отображение $C(Q) \rightarrow Y$ и $\tau(g)=y$. Так как $E_{1} \in \mathcal{E}$ по лемме 3 , найдется $g_{0} \in C(Q)$ : $\left\|g-g_{0}\right\|=\rho\left(g, E_{1}\right)=\varphi\left(g-g_{0}\right)$, а потому $\varphi \in P_{C}$. Итак, показано, что $P_{Y} \subset P_{C(Q)}$. По теореме Бишопа-Фелпса [13, теорема 1, c. 9$] P_{Y}$ всюду плотно в $L=\left(C(Q) / E_{1}\right)^{*}$ и лемма доказана.

Tеорема 3. Пусть $Q=\bar{Q}_{0}, \quad E \subset C(Q), \operatorname{dim} E=+\infty, \quad 1 \in E, C(Q) / E-$ пространство Линденштраусса. Тогда $E$ - не чебышевское подпространство.

ДоказАтельство. Теорема очевидна, если $E \notin \mathcal{E}$ или если $E^{\perp}$ содержит меры, носитель которых конечен. Поэтому будем предполагать, что $E \in \mathcal{E}$ и что $\operatorname{supp} \nu$ бесконечен для любой меры $\nu \in E^{\perp}$. Допустим, что найдется $q_{0} \in Q_{0}, q_{0} \notin \operatorname{supp} \varphi \forall \varphi \in$ $\partial B_{E^{\perp}}$. Так как по теореме Крейна-Мильмана [5, теорема 4, c. 477] $B_{E^{\perp}}=\overline{\mathrm{co}}\left(\partial B_{E^{\perp}}\right)$, то $Q_{0} \notin \operatorname{supp} \varphi \forall \varphi \in E^{\perp}$. Пусть $g \in C(Q),\left.g\right|_{\operatorname{supp} \varphi}=0 \forall \varphi \in E^{\perp}$. Тогда $g \in E$ и по лемме C $E$ - не чебьшевское подпространство. Следовательно, далее считаем, что $Q_{0} \subset \bigcup_{\varphi \in \partial B_{E} \perp} \operatorname{supp} \varphi$.

Предположим теперь, что для некоторого $\varphi_{0} \in \partial B_{E^{\perp}}$ носитель $\operatorname{supp} \varphi_{0}$ содержит две различные точки $q_{1}, q_{2} \in Q_{0}$. Определим функцию

$$
\tilde{g}(q)= \begin{cases}0, & q \neq q_{1}, q_{2} \\ \alpha_{1}, & q=q_{1} \\ \alpha_{2}, & q=q_{2}\end{cases}
$$

где $\alpha_{1} \neq 0, \alpha_{2} \neq 0$ подобраны так, чтобы $\varphi_{0}(\tilde{g})=0$. Тогда $\tilde{g} \in E$, поскольку $\varphi(\tilde{g})=$ $0 \forall \partial B_{E^{\perp}}$. Пусть $L=\left\{\varphi \in E^{\perp}: \varphi\left\{q_{1}\right\}=\varphi\left\{q_{2}\right\}=0\right\}$. Тогда $L-w^{*}$-замкнутое пространство в $E^{\perp}, \operatorname{dim} E^{\perp} / L=2$. По лемме 5 можно найти $\varphi \in P_{C} \cap L$, и так как $\tilde{g} \in E,\left.\tilde{g}\right|_{\operatorname{supp} \varphi}=0$, то $E$-не чебышевскоеподпространство по лемме С. Следовательно, нужно анализировать случай, когда $\forall \varphi \in \partial B_{E^{\perp}}$ носитель $\operatorname{supp} \varphi$ содержит не более одной точки $q \in Q_{0}$.

Возьмем $q_{1}, q_{2} \in Q_{0}$ и пусть $\varphi_{i} \in \partial B_{E^{\perp}}, q_{i} \in \operatorname{supp} \varphi_{i}, i=1,2$. Определим на $E^{\perp}$ $w^{*}$-непрерьвные функционалы $\gamma_{i}: \gamma_{i}(\varphi)=\varphi\left\{q_{1}\right\}, i=1,2$, и пусть $L=\left\{\varphi \in E^{\perp}\right.$ : $\left.\gamma_{i}(\varphi)=0, i=1,2\right\}-w^{*}$-замкнутое подпространство $E^{\perp}$. Возьмем $\varphi \in E^{\perp},\|\varphi\|=1$. По теореме Крейна-Мильмана [5, теорема 4, с. 477]

$$
\varphi=w^{*}-\lim \left(\alpha_{n}^{1} \varphi_{1}+\alpha_{n}^{2} \varphi_{2}+\sum_{i=3}^{r_{n}} \alpha_{n}^{i} \varphi_{i}\right), \quad \text { где } \quad \varphi_{n}^{i} \in \partial B_{E^{\perp}} \sum_{i=3}^{r_{n}}\left|\alpha_{n}^{i}\right|=1 .
$$


Считая, что $\alpha_{n}^{1} \rightarrow \alpha_{1}, \alpha_{n}^{2} \rightarrow \alpha_{2}$, получим, что $\varphi=\alpha_{1} \varphi_{1}+\alpha_{2} \varphi_{2}+\psi$, где $\psi \in L$. При этом, учитьвая замечание 1 , получаем

$$
\left\|\alpha_{n}^{1} \varphi_{1}+\alpha_{n}^{2} \varphi_{2}+\sum_{i=3}^{r_{n}} \alpha_{n}^{i} \varphi_{i}\right\|=1, \quad n=1,2, \ldots,
$$

и потому $\left|\alpha_{1}\right|+\left|\alpha_{2}\right|+\|\psi\| \leq 1$. С другой стороны, $\|\varphi\|=1 \leq\left|\alpha_{1}\right|+\left|\alpha_{2}\right|+\|\psi\|$. Поэтому $\|\psi\|=1-\left|\alpha_{1}\right|-\left|\alpha_{2}\right|$. Значит, если $\varphi \in E^{\perp}, \varphi=\alpha_{1} \varphi_{1}+\alpha_{2} \varphi_{2}+\psi, \psi \in L$,то $\|\varphi\|=\left|\alpha_{1}\right|+\left|\alpha_{2}\right|+\|\psi\|$.

Определим на $E^{\perp} w^{*}$-непрерывный функционал $\Gamma: \Gamma(\varphi)=\gamma_{1}(\varphi) / \gamma_{1}\left(\varphi_{1}\right), \varphi \in E^{\perp}$, и убедимся, что $\beta_{\delta_{q_{2}}}^{+}(\Gamma) \neq \beta_{\delta_{q_{2}}}^{-}(\Gamma)$. Если $\varphi \in E^{\perp},\|\varphi\|=1, \varphi=\alpha_{1} \varphi_{1}+\alpha_{2} \varphi_{2}+\psi, \psi \in L$, то $1-\Gamma(\varphi)=1-\alpha_{1}, \varphi\left\{q_{2}\right\}=\alpha_{2} \varphi_{2}\left\{q_{2}\right\}$. Следовательно, неравенство $1-\Gamma(\varphi) \leq 2\left|\varphi\left\{q_{2}\right\}\right|$ приобретает вид $1-\alpha_{1} \leq 2\left|\alpha_{2}\right| \cdot\left|\varphi_{2}\left\{q_{2}\right\}\right| \leq\left|\alpha_{2}\right|$. Ясно, что последнее возможно лишь, если $\left|\varphi_{2}\left\{q_{2}\right\}\right|=1 / 2$ и $\left|\alpha_{1}\right|+\left|\alpha_{2}\right|=1$. В этом случае $\varphi=\alpha_{1} \varphi_{1}+\alpha_{2} \varphi_{2}$ и

$$
\frac{1-\Gamma(\varphi)}{\left|\varphi\left\{q_{2}\right\}\right|}=\frac{1-\alpha_{1}}{\frac{1}{2}\left(1-\left|\alpha_{1}\right|\right)} \geq 2 \text {. }
$$

Значит, $\beta_{\delta_{q_{2}}}^{+}(\Gamma)=+1$. Аналогичные рассуждения показьвают, что $\beta_{\delta_{q_{2}}}^{-}(\Gamma)=-1$. Следовательно, не вьполнено условие 1 ) теоремы 2 и $E$ - не чебьшевское подпространство. Теорема доказана.

\section{СПИСОК ЦИТИРОВАННОЙ ЛИТЕРАТУРЫ}

[1] Lacay H. E. The isometric theory of classical Banach spaces. Berlin: Springer, 1974.

[2] Шашкин Ю.А. Выпуклые множества, экстремальные точки, симплексы // Итоги науки и техники. Матем. анализ. 1973. Т. 11. С. 5-50.

[3] Данфорд Н., Шварц Дж. Т. Линейные операторы. Т. 1. М.: Мир, 1962.

[4] Устинов Г. М. О подпространствах существования в $C(Q, X)$ // Исслед. по функцион. анализу и топологии. Свердловск, 1990. С. 127-130.

[5] Гаркави А. Л. О наилучшем приближении элементами бесконечномерных подпространств одного класса // Матем. сб. 1963. Т. 62. №1. С. 104-120.

[6] Effros E. G. Structure in simplexes. II // J. Funct. Anal. 1967. V. 1. № 4. P. 379-391.

[7] Lazar A. J. Spaces of affine continuous functions on simplexes // Trans. Amer. Math. Soc. 1968. V. 134. № 3. P. 503-525.

[8] Mach J. Continuity properties of Chebyshev centers // J. Approx. Theory. 1980. V. 29. № 3. P. 223-230.

[9] Колмогоров А. Н., Фомин С. В. Элементы теории функций и функционального анализа. М.: Наука, 1972.

[10] Phelps R. R. Uniqueness of Hahn-Banach extensions and unique best approximation // Trans. Amer. Math. Soc. 1960. V. 95. № 2. P. 238-255.

[11] Власов Л. П. Характеристика обобщенных и классических элементов наилучшего приближения относительно подпространств конечной коразмерности // Матем. заметки. 1980. Т. 28. № 5. C. 707-715.

[12] Устинов Г. М. О подпространствах единственности в пространствах абстрактных непрерывных функций. Препринт/АН СССР. УрО. ИММ. Свердловск, 1987.

[13] Дистель Дж. Геометрия банаховых пространств. Киев: Вища школа, 1980.

Уральский государственный технический университет им. С. М. Кирбы

Поступило г. Екатеринбург 28.10 .96

Исправленный вариант 03.02 .98 